This is the accepted manuscript made available via CHORUS. The article has been published as:

\title{
Strongly Spin-Orbit Coupled Two-Dimensional Electron Gas Emerging near the Surface of Polar Semiconductors
}

M. Sakano, M. S. Bahramy, A. Katayama, T. Shimojima, H. Murakawa, Y. Kaneko, W. Malaeb, S. Shin, K. Ono, H. Kumigashira, R. Arita, N. Nagaosa, H. Y. Hwang, Y. Tokura, and K. Ishizaka

Phys. Rev. Lett. 110, 107204 - Published 7 March 2013

DOI: 10.1103/PhysRevLett.110.107204 


\title{
Strongly spin-orbit coupled two-dimensional electron gas emerging near the surface of polar semiconductors
}

\author{
M. Sakano, ${ }^{1}$ M. S. Bahramy ${ }^{2}$ A. Katayama, ${ }^{1}$ T. Shimojima, ${ }^{1}$ H. Murakawa, ${ }^{2}$ \\ Y. Kaneko, ${ }^{2}$ W. Malaeb, ${ }^{3,4}$ S. Shin, ${ }^{3,4}$ K. Ono, ${ }^{5}$ H. Kumigashira, ${ }^{5}$ R. \\ Arita, ${ }^{1,2,6}$ N. Nagaosa, ${ }^{1,2}$ H. Y. Hwang, ${ }^{2,7}$ Y. Tokura, ${ }^{1,2}$ and K. Ishizaka ${ }^{1,6}$ \\ ${ }^{1}$ Department of Applied Physics, The University of Tokyo, Tokyo 113-8656, Japan \\ ${ }^{2}$ Correlated Electron Research Group (CERG), \\ RIKEN Advanced Science Institute, Wako, Saitama 351-0198, Japan \\ ${ }^{3}$ Institute of Solid State Physics, The University of Tokyo, Kashiwa, Chiba, 227-8581, Japan \\ ${ }^{4}$ CREST, JST, Tokyo 102-0075, Japan \\ ${ }^{5}$ Institute of Materials Structure Science, \\ High Energy Accelerator Research Organization (KEK), Tsukuba, Ibaraki 305-0801, Japan \\ ${ }^{6}$ JST, PRESTO, Kawaguchi, Saitama 332-0012, Japan \\ ${ }^{7}$ Department of Applied Physics and Stanford Institute for Materials and Energy Science, \\ Stanford University, Stanford, California 94305, USA
}

\begin{abstract}
We investigate the two-dimensional (2D) highly spin-polarized electron accumulation layers commonly appearing near the surface of $n$-type polar semiconductors $\mathrm{BiTeX}(\mathrm{X}=\mathrm{I}, \mathrm{Br}$, and $\mathrm{Cl})$ by angular-resolved photoemission spectroscopy. Due to the polarity and the strong spin-orbit interaction built in the bulk atomic configurations, the quantized conduction-band subbands show giant Rashba-type spin-splitting. The characteristic 2D confinement effect is clearly observed also in the valence-bands down to the binding energy of $4 \mathrm{eV}$. The X-dependent Rashba spin-orbit coupling is directly estimated from the observed spin-split subbands, which roughly scales with the inverse of the band-gap size in BiTeX.
\end{abstract}


In a nonmagnetic solid compound, spatial-inversion and time-reversal symmetries require the degeneracy of an electron's spin, which can be lifted in a noncentrosymmetric system by introducing spin-orbit interaction (SOI). The Bychkov-Rashba (BR) effect describes such SOI in an inversion-symmetry broken system, by simply considering a two-dimensional (2D) electron gas with an electric field applied along its perpendicular direction [1]. The Hamiltonian of the $\mathrm{BR}$ model is given by $H_{\mathrm{BR}}=\lambda \vec{\sigma} \cdot\left(\vec{E}_{z} \times \vec{k}\right)$, where $\lambda$ is the coupling constant reflecting the SOI, $\vec{\sigma}$ and $\vec{k}$ are the spin and momentum of electrons, and $\vec{E}_{z}$ is the applied electric field. It gives an effective magnetic field acting on the electron spin $\vec{\sigma}$, with orientation normal to $\vec{E}_{z}$ and $\vec{k}$. The energy dispersion is characterized by the $k$-linear splitting of the free electron parabolic band dispersion as denoted by $E^{ \pm}(\vec{k})=\hbar^{2} \vec{k}^{2} / 2 m^{*} \pm \alpha_{\mathrm{R}}|\vec{k}|$, where $m^{*}$ is the effective mass of the electron and $\alpha_{\mathrm{R}}$ gives the degree of Rashba-type spin-splitting i.e. Rashba parameter. Until now, such asymmetry-induced spin-splitting has been actively investigated in semiconductor heterostructures utilizing GaAs systems [2]. More recently, attempts to induce spin-splitting by applying the electric field in centrosymmetric heavyelement oxide semiconductors, such as $\mathrm{KTaO}_{3}$, are also reported [3]. The spin-splittings in these systems have remained small compared to the thermal energy at room temperature, nevertheless, due to the weak SOI and/or small asymmetric $\vec{E}_{z}$ that can be applied by such field effects.

Previously we reported the giant spin-splitting realized in the bulk of a polar semiconductor, BiTeI [4-7]. Its peculiar polar crystal structure leads to a bulk-induced Rashba-type SOI, similarly as originally discussed in wurzite semiconductor materials [8]. The Rashba parameter $\alpha_{\mathrm{R}}$ for BiTeI is found to be as large as $3.5 \mathrm{eV} \AA$, which is comparable to the largest value reported thus far in a surface alloyed metal $\mathrm{Bi} / \mathrm{Ag}(111)$ system [9]. By utilizing such a large Rashba-type spin-splitting nature built in the bulk semiconductor material, one may obtain a 2D electron system under a strong Rashba field near its surface and interface, as highly desired from the viewpoint of developing various spintronic functions $[10,11]$ and multi-functional interfaces [12]. In this Letter, we investigate the near-surface regions of the extended families of polar semiconductors $\mathrm{BiTeX}(\mathrm{X}=\mathrm{I}, \mathrm{Br}$, and $\mathrm{Cl}$ ) by means of highresolution angular-resolved photoemission spectroscopy (ARPES). Using first-principles calculations combined with the Poisson-Schrödinger (PS) method [13], we precisely characterize the $2 \mathrm{D}$ confinement effect on electronic structures including both conduction- and valencebands, emerging as a consequence of charge accumulation at the surfaces. 
Single crystals of BiTeI were grown by the Bridgman method [4], whereas those of BiTeBr and $\mathrm{BiTeCl}$ were grown by the chemical vapor transport method. Laser-ARPES was performed using a vacuum ultraviolet laser $(h \nu=6.994 \mathrm{eV})$ and a VG-Scienta R4000 electron analyzer [14] with a total resolution energy of $4 \mathrm{meV}$. $h \nu$-dependent ARPES measurement $(h \nu=60-84 \mathrm{eV})$ was performed by using the VG-Scienta SES2002 electron analyzer at BL28 in Photon Factory, KEK, with a total resolution energy of 20 - $30 \mathrm{meV}$. Samples were cleaved in situ at around room temperature and measured at 10-15 K. Electronic-structure calculations for BiTeX were carried out within the context of density functional theory using the full-potential augmented plane-wave plus local orbital (APW-LO) method [4]. To simulate the effect of band bending, a 60 layer tight-binding supercell was constructed by downfolding the APW-LO Hamiltonian, using maximally localized Wannier functions [1517]. The band bending potential was then obtained by solving the coupled PS equation [13], assuming static dielectric constants 15,10 , and 6.5 for $\mathrm{X}=\mathrm{I}, \mathrm{Br}$, and $\mathrm{Cl}$, respectively.

The crystal structures of BiTeX are shown in Figs. 1(a) (X = I and Br; space group P3m1) and $1(\mathrm{~b})\left(\mathrm{X}=\mathrm{Cl} ; P 6_{3} m c\right)$. They are characterized by the alternative stacking of Bi-, Te- and X-layers, which gives rise to the polarity along the stacking axis. In contrast to the previous report indicating the disorder of Te and $\mathrm{Br}$ sites for BiTeBr [18], the single crystalline BiTeBr we obtained was confirmed to possess the well-defined stacking of Teand Br- triangular lattices by x-ray diffraction measurements.

The calculated band structures for BiTeI and BiTeBr (BiTeCl) as shown in Fig. 1(f)-1(h) are composed of $6(12)$ conduction bands above the Fermi level $\left(E_{\mathrm{F}}\right)$ and $12(24)$ valence bands below $E_{\mathrm{F}}$. The conduction bands for BiTeX are predominantly composed of $\mathrm{Bi} 6 p$ components. The valence bands, on the other hand, show a systematic X-dependence, as seen by the lower half of them moving toward deeper binding energy $\left(E_{\mathrm{B}}\right)$ upon increasing the electronegativity of $\mathrm{X}$ from $\mathrm{I}, \mathrm{Br}$, to $\mathrm{Cl}$. This indicates that the lower set of the valence bands is mainly dominated by $\mathrm{X}$ ions, whereas the upper ones primarily reflect the Te $5 p$ character. The resistivity for each sample, as shown in Fig. 1(e), indicates metallic behavior down to $2 \mathrm{~K}$. The electron densities of $\mathrm{X}=\mathrm{I}, \mathrm{Br}$, and $\mathrm{Cl}$ samples are estimated from the Hall coefficient to be $n_{\text {bulk }}=4 \times 10^{19}, 4 \times 10^{18}$, and $2 \times 10^{18} \mathrm{~cm}^{-3}$, respectively, indicating that they all behave as $n$-type degenerate semiconductors due to slight nonstoichiometry. From the band calculation, $E_{\mathrm{F}}$ of the bulk state corresponding to these carrier concentrations should be located at $142 \mathrm{meV}(\mathrm{X}=\mathrm{I}), 27 \mathrm{meV}(\mathrm{X}=\mathrm{Br})$, and $5 \mathrm{meV}(\mathrm{X}=\mathrm{Cl})$ above their 
respective conduction band minimums (CBMs).

Figure 2(a) shows the laser-ARPES image for BiTeBr, recorded along the $\Gamma-\mathrm{K}\left(k_{y}\right)$ direction. The CBM reaches $E_{\mathrm{B}} \sim 0.47 \mathrm{eV}$, with the remarkable Rashba-type band splitting of up to $\sim 0.2 \mathrm{eV}$. The observed $\mathrm{CBM}$ is quite deep below $E_{\mathrm{F}}$, considering the low carrier density of the bulk BiTeBr with $E_{\mathrm{F}}-E_{\mathrm{CBM}}=27 \mathrm{meV}$. One can also notice that there are two additional sets of bands at shallower energies with CBMs at around $E_{\mathrm{B}}=0.2$ and $0.1 \mathrm{eV}$, thus forming a ladder of three Rashba-split subbands. These clearly indicate that downward band bending occurs near the surface of BiTeBr, forming an $n$-type accumulation layer. The conduction electrons confined in such 2D accumulation layers are known to form quantized conduction subbands $[13,19,20]$. In the present case of BiTeBr, all the subbands clearly distinguished indicate the giant Rashba-type splittings, thus offering direct evidence of a 2D spin-polarized electron system dominated by the huge SOI field.

To grasp the topology and shape of these spin-split subbands, the constant energy crosssectional views of the band dispersions are shown in Fig. 2(b). At $E_{\mathrm{B}}=0$, i.e. $E_{\mathrm{F}}$, six Fermi surfaces (FSs) corresponding to inner and outer FSs for the $n=1 \sim 3$ subbands are observed. The large ones, namely the outer and inner $n=1 \mathrm{FSs}$, are strongly distorted, while the cross-sections of the bands become smaller and more isotropic at deeper $E_{\mathrm{B}}$. The band crossing point is also clearly distinguished as a sharp point appearing at the zone center for some specific $E_{\mathrm{B}}$ (e.g. $160 \mathrm{meV}$ for the $n=2$ subband). To discuss the realized $2 \mathrm{D}$ electronic structure, we introduce the PS method to calculate the subband structures by taking into account the band bending potential and 2D confinement at the accumulation layer [13]. Here, we fix the bulk carrier density and calculate the depth $(z)$-dependent energy $V(z)$ and electron density $n(z)$, which most accurately reproduce the observed subbands configuration [see Fig. 3(b)]. $V(z)$ and $n(z)$ are shown in Figs. 2(c) and (d), which indicate that the electron accumulation layer of $\sim 1 \mathrm{~nm}$ thickness is formed at the near-surface region of BiTeBr.

The systematic results of laser-ARPES and corresponding PS simulation for BiTeX are shown in Fig. 3. The experimentally obtained subband dispersions along $k_{y}$ directions are shown in Figs. 3(a)-3(c), whereas their FSs are shown in Figs. 3(g)-(i). Markers showing the peak positions of ARPES intensity estimated from the energy distribution curves and momentum distribution curves are overlaid on the images. BiTeI (BiTeBr and BiTeCl) exhibits two (three) sets of Rashba-type spin-split conduction subbands. Subbands of $n=1$, 
2, and 3 are depicted by red, blue, and green markers. The black triangle markers in Fig. $3(\mathrm{~g})$ show the possible bulk contribution (see supplemental material). The CBMs of the deepest subband $(n=1)$ are located at $E_{\mathrm{B}} \sim 0.27,0.47$, and $0.41 \mathrm{eV}$ for $\mathrm{X}=\mathrm{I}, \mathrm{Br}$, and $\mathrm{Cl}$, respectively, which are all much deeper when compared to the respective bulk $E_{\mathrm{F}}-E_{\mathrm{CBM}}$. The 2D electron density at the accumulation layer can be estimated experimentally by counting the areas of all the observed FSs in Figs. $3(\mathrm{~g})-3(\mathrm{i}) ; n_{2 \mathrm{D}}=3.0 \times 10^{13}, 7.1 \times 10^{13}$, and $4.6 \times 10^{13} \mathrm{~cm}^{-2}$ for $\mathrm{X}=\mathrm{I}, \mathrm{Br}$, and $\mathrm{Cl}$, respectively. The $V(z)$ and $n(z)$ which most well reproduce the observed subband positions for BiTeX are given in Figs. 2(c) and 2(d). The calculated subband dispersions in Figs. 3(d)-3(f) agree well with the ARPES data, including the group velocities and spin-splittings of the respective subbands. By comparing the FSs [Figs. 3(g)-3(l)], we can also demonstrate that the PS method quantifies their sizes and shapes ( $k_{x^{-}}-k_{y}$ anisotropy) as well. Note that the $n=1$ inner and outer FSs get closer at $k_{x}(\Gamma-\mathrm{M})$ direction as compared to $k_{y}(\Gamma-\mathrm{K})$ direction. This behavior can be qualitatively understood by looking at the bulk band dispersions near the CBM. For BiTeBr as shown in Fig. $1(\mathrm{~g})$, for example, the spin-split band dispersions along the $\mathrm{A}-\mathrm{H}\left(k_{y}\right)$ direction remain almost parallel, whereas those along A-L $\left(k_{x}\right)$ cross each other at $\sim 0.8 \mathrm{eV}$ above the CBM. This is due to the higher-order term of SOI reflecting the underlying crystal symmetry, namely the trigonal warping effect [21], giving rise to the observed deformation of the FSs at large $k$ vectors. Such warping effect accompanies the spin polarization away from the BR model, appearing as the out-of-plane spin component $\sigma_{z}$ along $k_{y}$ direction. According to the calculation, the ratio of the out-of-plane to in-plane spin component reaches $79 \%$ along $k_{y}$ at $E-E_{\mathrm{CBM}}=450 \mathrm{meV}$ in BiTeI (see supplemental material).

To quantitatively evaluate the spin-splittings, we estimate the Rashba-parameter $\alpha_{\mathrm{R}}$ for BiTeX. Here we calculate $\alpha_{\mathrm{R}}$ by using the momentum offset at CBMs $\left(k_{0}\right)$ and the Rashba energy $\left(E_{\mathrm{R}}\right)$, as depicted in Fig. $3(\mathrm{~d})$. They are related by $E_{\mathrm{R}}=\hbar^{2} k_{0}{ }^{2} / 2 m^{*}$ and $\alpha_{\mathrm{R}}=2 E_{\mathrm{R}} / k_{0}$ in the $2 \mathrm{D}$ free-electron $\mathrm{BR}$ model. Estimated values for $E_{\mathrm{R}}, k_{0}$ and $\alpha_{\mathrm{R}}$ are shown in Table I. There is no clear $n$-dependence in the obtained $\alpha_{\mathrm{R}}$. Regarding the X-dependence, the experimentally obtained values of $\alpha_{\mathrm{R}}$ for BiTeI (3.9 - $\left.4.3 \mathrm{eV} \AA\right)$ are significantly larger when compared to $\mathrm{BiTeBr}(2.0$ - $2.1 \mathrm{eV} \AA)$ and $\mathrm{BiTeCl}(1.7-2.2 \mathrm{eV} \AA)$. Similar values and tendencies are also indicated in the series of $\alpha_{\mathrm{R}}^{c}$ estimated from the calculated subbands. Nearly $n$-independent behavior of $\alpha_{\mathrm{R}}$ obtained for BiTeX is in contrast to the case of centrosymmetric $\mathrm{Bi}_{2} \mathrm{Se}_{3}$, where the Rashba-type spin splitting of the conduction band is considered to 
be induced by the band bending effect [22]. This indicates that the spin-splitting of BiTeX predominantly originates from the bulk polar crystal structure, whose net effect on each layer is much larger than that of band bending.

The huge spin-splitting of the CB in bulk BiTeI has been successfully explained by means of $k \cdot p$ perturbation theory [5]. According to this model, the SOI-induced band splitting near the $E_{\mathrm{F}}$, i.e. $\mathrm{CBM}$ and valence band maximum $(\mathrm{VBM})$, is strongly affected by the unusual electronic structures around its band gap. In BiTeX, the VBM (CBM) is commonly composed of Te $5 p$ (Bi $6 p$ ) orbitals. The band-gap size for BiTeX, on the other hand, is known to depend on $\mathrm{X}$ anions: The minimum gaps realized in BiTeX are found to be 0.36, 0.6, and $0.7 \mathrm{eV}$ for $\mathrm{X}=\mathrm{I}, \mathrm{Br}$, and $\mathrm{Cl}$, respectively, from optical studies [23]. The narrow energy separation between CBM and VBM, combined with the large atomic SOI of Bi, enhances the spin splitting via the second order perturbative $k \cdot p$ Hamiltonian [5]. Considering these aspects, the X-dependent spin-splitting in BiTeX can be primarily understood to follow the inverse of the band-gap size, as observed. The huge spin-splitting commonly emerging in BiTeX should drive the development of high-efficient spintronic devices, as practically predicted by a recent calculation [24].

Finally, we investigate the effect of the 2D electron accumulation in a wider energy range, including the valence bands. The second-derivative ARPES images for BiTeI taken along $k_{x}$ direction are shown in Figs. 4(a)-(c) for $h \nu=64,72$, and $80 \mathrm{eV}$, which correspond to $k_{z}=\pi / c, 1.51 \pi / c$, and $0(2 \pi / c)$. The conduction subbands with $E_{\mathrm{CBM}}=0.3 \mathrm{eV}$ (red) and $0.2 \mathrm{eV}$ (blue) for $n=1$ and 2, similar to the laser-ARPES result in Fig. 3(a), are commonly observed, thus indicating their 2D characters. Regarding the valence bands, the peak positions of the ARPES images which are $k_{z}$-dependent ( $k_{z}$-independent) are carefully separated and plotted by green (red and blue) markers. The green markers correspond to the bulk band dispersions, which agree well with those previously obtained by the bulk-sensitive soft x-ray ARPES [6]. Because of the surface sensitivity, however, the presently observed ARPES image tends to be broadened due to the $k_{z}$ summation. On the other hand, the $k_{z}$-independent 2D band dispersions can be clearly observed, whose shapes are strikingly different from any $k_{z}$ components of the bulk band dispersions.

To account for the observed 2D structures, we calculated the near-surface electronic structure of BiTeI in a wider energy-range. Figure 4(d) shows the electronic structure formed in the topmost Te-Bi-I trilayer (TL), whereas Fig. 4(e) indicates the projection 
of all TLs into the bulk. These calculated data show the ladders of spin-split quantized subbands ( $n=1$ and 2 ) formed near the surface (1TL), extending in a wide energy region of the valence bands. By comparing with this PS calculation, the red (blue) markers in the ARPES image can be successfully assigned to the $n=1(n=2)$ subbands of the valence bands (the deepest one could not be clearly distinguished experimentally). It thus indicates that not only conduction-bands but also valence-bands, that are deeply bound to $\sim 4 \mathrm{eV}$ below $E_{\mathrm{F}}$, are well separated from the bulk and quantized near the surface as a consequence of $2 \mathrm{D}$ electronic confinement.

The present finding of 2D strong Rashba-field electron system, standing separately from bulk in a wide-energy range, thus reveals the potential of polar BiTeX for designing various interfaces with strong SOI. It is also worth noting that the Rashba parameter and the bulk chemical-potential are tunable in this system by chemical substitution and doping. The origin and mechanism of the near-surface band-bending for BiTeX, nevertheless, are yet unclear. Very recently, several studies report that accumulation (depletion) tends to occur at the Te- (X-) terminated surface [25-27]. It will be interesting to elucidate the near-surface charge accumulation in polar semiconductors from the viewpoint of the bulk (carrier density, band structure, polarity, etc.) and surface/interface properties, for further engineering of 2D strong SOI electron systems.

In summary, we investigated the electronic structure near the surface of polar semiconductors BiTeX. The quantized subbands with huge Rashba-type splitting were commonly observed in BiTeX, indicating the 2D electron accumulation layer with strong SOI robustly realized owing to the peculiar bulk structure. The observed X-dependence of the Rashba parameter shows the importance of the band-gap size in designing the SOI field near $E_{\mathrm{F}}$. Together with the PS calculation, the 2D quantization was found to occur in a wide range of energy, including the valence bands deeply bound at several eV below $E_{\mathrm{F}}$.

This work was partly supported by JST, PRESTO, Grant-In-Aid for Japan, and by Funding Program for World-Leading Innovation R \& D on Science and Technology (FIRST). H.Y.H. acknowledges support by the Department of Energy, Office of Basic Energy Sciences, Materials Sciences and Engineering Division, under contract DE-AC02-76SF00515.

[1] Yu. A. Bychkov and E. I. Rashba, JETP Lett. 39, 78 (1984). 
[2] J. Nitta, T. Akazaki, H. Takayanagi, and T. Enoki, Phys. Rev. Lett. 78, 1335 (1997).

[3] H. Nakamura and T. Kimura, Phys. Rev. B. 80, 121308(R) (2009).

[4] K. Ishizaka, M. S. Bahramy, H. Murakawa, M. Sakano, T. Shimojima, T. Sonobe, K. Koizumi, S. Shin, H. Miyahara, A. Kimura, K. Miyamoto, T. Okuda, H. Namatame, M. Taniguchi, R. Arita, N. Nagaosa, K. Kobayashi, Y. Murakami, R. Kumai, Y. Kaneko, Y. Onose, and Y. Tokura, Nature Mater. 10, 521 (2011).

[5] M. S. Bahramy, R. Arita, and N. Nagaosa, Phys. Rev. B 84, 041202(R) (2011).

[6] M. Sakano, J. Miyawaki, A. Chainani, Y. Takata, T. Sonobe, T. Shimojima, M. Oura, S. Shin, M. S. Bahramy, R. Arita, N. Nagaosa, H. Murakawa, Y. Kaneko, Y. Tokura, and K. Ishizaka, Phys. Rev. B 86, 085204 (2012).

[7] J. S. Lee, G. A. H. Schober, M. S. Bahramy, H. Murakawa, Y. Onose, R. Arita, N. Nagaosa, and Y. Tokura, Phys. Rev. Lett. 107, 117401 (2011).

[8] E. I. Rashba, Fiz. Tverd. Tela (Leningrad) 2, 1224 (1960) [Sov. Phys. Solid State 2, 1109 (1960)].

[9] C. R. Ast, J. Henk, A. Ernst, L. Moreschini, M. C. Falub, D. Pacil'e, P. Bruno, K. Kern, and M. Grioni, Phys. Rev. Lett. 98186807 (2007).

[10] S. Datta and B. Das, Appl. Phys. Lett. 56, 665 (1990).

[11] J. Sinova, D. Culcer, Q. Niu, N. A. Sinitsyn, T. Jungwirth, and A. H. MacDonald Phys. Rev. Lett. 92, 126603 (2004).

[12] H. Y. Hwang, Y. Iwasa, M. Kawasaki, B. Keimer, N. Nagaosa, and Y. Tokura, Nature Mater. 11, 103 (2012).

[13] P. D. C. King, T. D. Veal, and C. F. McConville, Phys. Rev. B 77, 125305 (2008).

[14] T. Kiss, T. Shimojima, K. Ishizaka, A. Chainani, T. Togashi, T. Kanai, X.-Y. Wang, C.-T. Chen, S. Watanabe, and S. Shin, Rev. Sci. Instrum. 79, 023106 (2008).

[15] I. Souza, N. Marzari, and D. Vanderbilt, Phys. Rev. B 65, 035109 (2001).

[16] A. A. Mostofi, J. R. Yates, Y.-S. Lee, D. Vanderbilt, and N. Marzari, Comp. Phys. Commun. 178, 685-699 (2008).

[17] J. Kuneš, R. Arita, P. Wissgott, A. Toschi, H. Ikeda, and K. Held, Comp. Phys. Commun. 181, 1888-1895 (2010).

[18] A. V. Shevelkov, E. V. Dikarev, R. V. Shpanchenko, and B. A. Popovkin, J. Solid State Chem. 114, 379 (1995). 
[19] A. F. Santander-Syro, O. Copie, T. Kondo, F. Fortuna, S. Pailhès, R. Weht, X. G. Qiu, F. Bertran, A. Nicolaou, A. Taleb-Ibrahimi, P. Le Fèvre, G. Herranz, M. Bibes, N. Reyren, Y. Apertet, P. Lecoeur, A. Barthélémy, and M. J. Rozenberg, Nature 469, 189 (2011).

[20] W. Meevasana, P. D. C. King, R. H. He, S.-K. Mo, M. Hashimoto, A. Tamai, P. Songsiriritthigul, F. Baumberger, and Z-X. Shen, Nature Mater. 10, 114 (2011).

[21] M. S. Bahramy, B.-J. Yang, R. Arita, and N. Nagaosa, Nature Commun. 3, 679 (2012).

[22] M. S. Bahramy, P. D. C. King, A. de la Torre, J. Chang, M. Shi, L. Patthey, G. Balakrishnan, Ph. Hofmann, R. Arita, N. Nagaosa, and F. Baumberger Nature Commun. 3, 1159 (2012).

[23] J. S. Lee et al., unpublished.

[24] K. Tsutsui and S. Murakami., Phys. Rev. B 86, 115201 (2012).

[25] S. V. Eremeev, I. A. Nechaev, Yu. M. Koroteev, P. M. Echenique, and E. V. Chulkov, Phys. Rev. Lett. 108, 246802 (2012).

[26] A. Crepaldi, L. Moreschini, G. Autes̀, C. Tournier-Colletta, S. Moser, N. Virk, H. Berger, Ph. Bugnon, Y. J. Chang, K. Kern, A. Bostwick, E. Rotenberg, O. V. Yazyev, and M. Grioni, Phys. Rev. Lett. 109, 096803 (2012).

[27] G. Landolt, S. V. Eremeev, Y. M. Koroteev, B. Slomski, S. Muff, T. Neupert, M. Kobayashi, V. N. Strocov, T. Schmitt, Z. S. Aliev, M. B. Babanly, I. R. Amiraslanov, E. V. Chulkov, J. Osterwalder, and J. H. Dil, Phys. Rev. Lett. 109, 116403 (2012). 
FIG. 1: (Color online) Crystal structures of BiTeI, BiTeBr (a) and BiTeCl (b). Brillouin zones of BiTeI, BiTeBr (c) and BiTeCl (d). (e) Temperature-dependent electrical resistivity of BiTeX. (f)-(h) Band dispersions of BiTeX obtained from first-principles calculations.

FIG. 2: (Color online) (a) Laser-ARPES spectral image of BiTeBr along the $k_{y}$ direction. (b) Constant energy cross-sectional views of the subband structure at intervals of $80 \mathrm{meV}$. (c),(d) Depth $(z)$-dependent potential energy $V(z)$ and electron density $n(z)$ of near-surface BiTeX calculated by the PS method.

FIG. 3: (Color online) (a)-(c) Laser-ARPES spectral images of BiTeX. The peak positions of ARPES intensity corresponding to $n=1,2$, and 3 subbands are plotted by red, blue, and green markers. (d)-(f) Corresponding subband structures obtained by PS method. (g)-(i) Intensity mappings at $E_{\mathrm{F}}$ obtained by laser-ARPES, which correspond to the 2D Fermi surfaces near the surface. Black triangle markers in (g) indicate the possible contributions of the bulk bands. (j)-(l) Corresponding Fermi surfaces calculated by PS method. z-dependent potential energy and electron density in obtained are shown in Figs. 2(c) and (d).

FIG. 4: (Color online) (a)-(c) Second-derivative ARPES images of BiTeI along the $k_{x}$ direction at $k_{z}=\pi / c, 1.51 \pi / c$, and $0(2 \pi / c)$, respectively. The peak positions of ARPES intensity corresponding to the $k_{z}$-dependent bulk bands ( $k_{z}$-independent subbands for $n=1$ and 2 ) are plotted by green (red and blue) markers. (d),(e) Electronic structures for the topmost Te-Bi-I trilayer and the projection of the electronic structures from all trilayers into the bulk, obtained by PS calculation. 
TABLE I: Rashba energy $E_{\mathrm{R}}(\mathrm{meV})$, momentum offset $k_{0}\left(10^{-3} \AA^{-1}\right)$, experimental and calculated Rashba parameters $\alpha_{\mathrm{R}}$ and $\alpha_{\mathrm{R}}^{c}(\mathrm{eV} \AA)$ of the $n$-th subband dispersions in BiTeX. Numbers in parentheses represent the respective range of error.

\begin{tabular}{ccccccccc}
\hline \hline & \multicolumn{2}{c}{ BiTeI } & \multicolumn{3}{c}{$\mathrm{BiTeBr}$} & \multicolumn{3}{c}{ BiTeCl } \\
\hline$n$ & 1 & 2 & 1 & 2 & 3 & 1 & 2 & 3 \\
\hline$E_{\mathrm{R}}$ & $108(13)$ & $92(13)$ & $42(10)$ & $37(7)$ & $35(8)$ & $25(10)$ & $17(6)$ & $20(6)$ \\
$k_{0}$ & $50(10)$ & $47(7)$ & $43(10)$ & $38(6)$ & $34(8)$ & $26(8)$ & $20(6)$ & $18(8)$ \\
$\alpha_{\mathrm{R}}$ & $4.3(9)$ & $3.9(8)$ & $2.0(7)$ & $2.0(5)$ & $2.1(7)$ & $1.9(10)$ & $1.7(8)$ & $2.2(12)$ \\
$\alpha_{\mathrm{R}}^{c}$ & 3.06 & 3.02 & 1.78 & 1.67 & 1.84 & 1.28 & 1.29 & 1.78 \\
\hline \hline
\end{tabular}




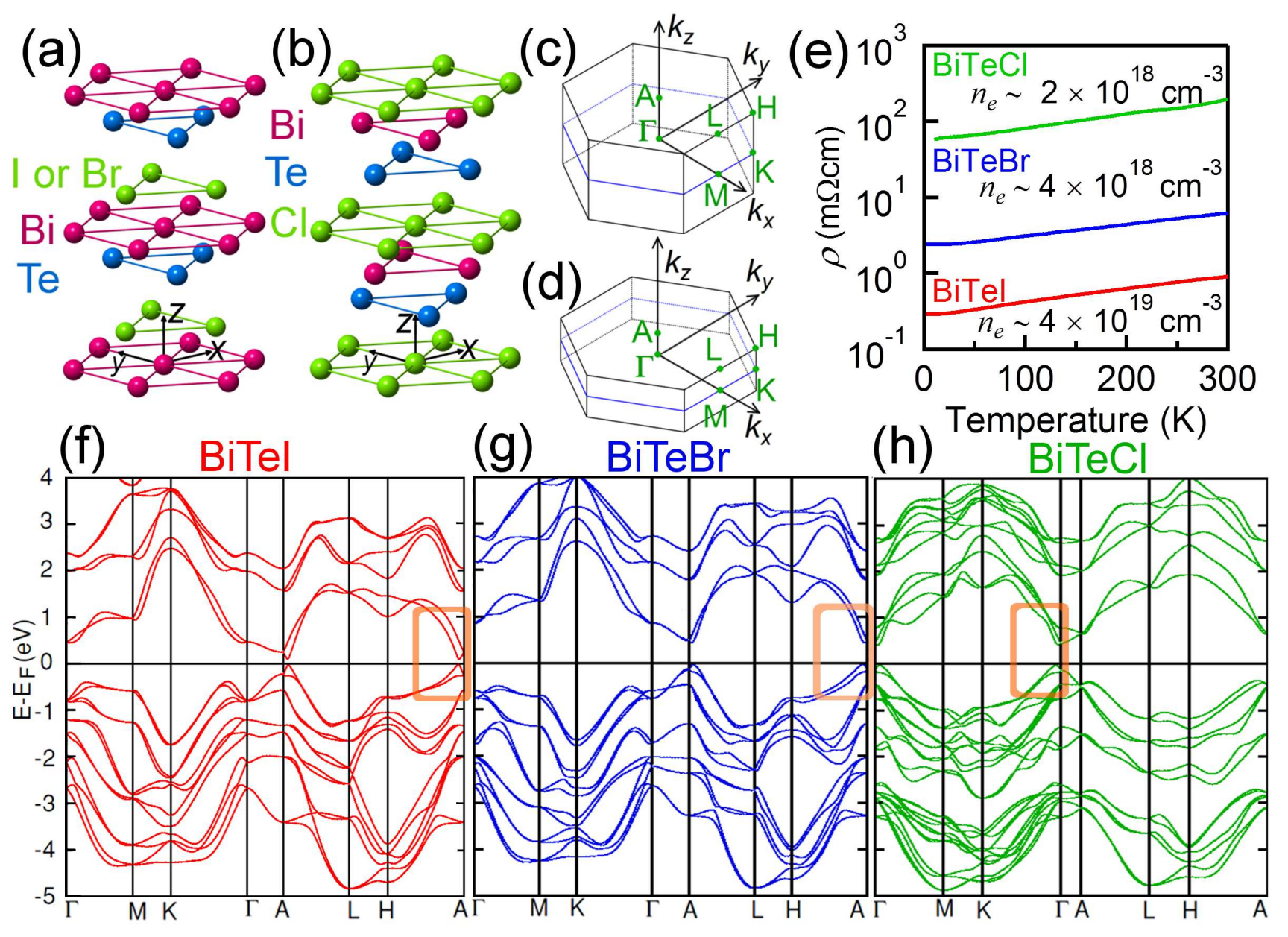

Figure 1 LY13668 $\quad$ 05Feb2013 


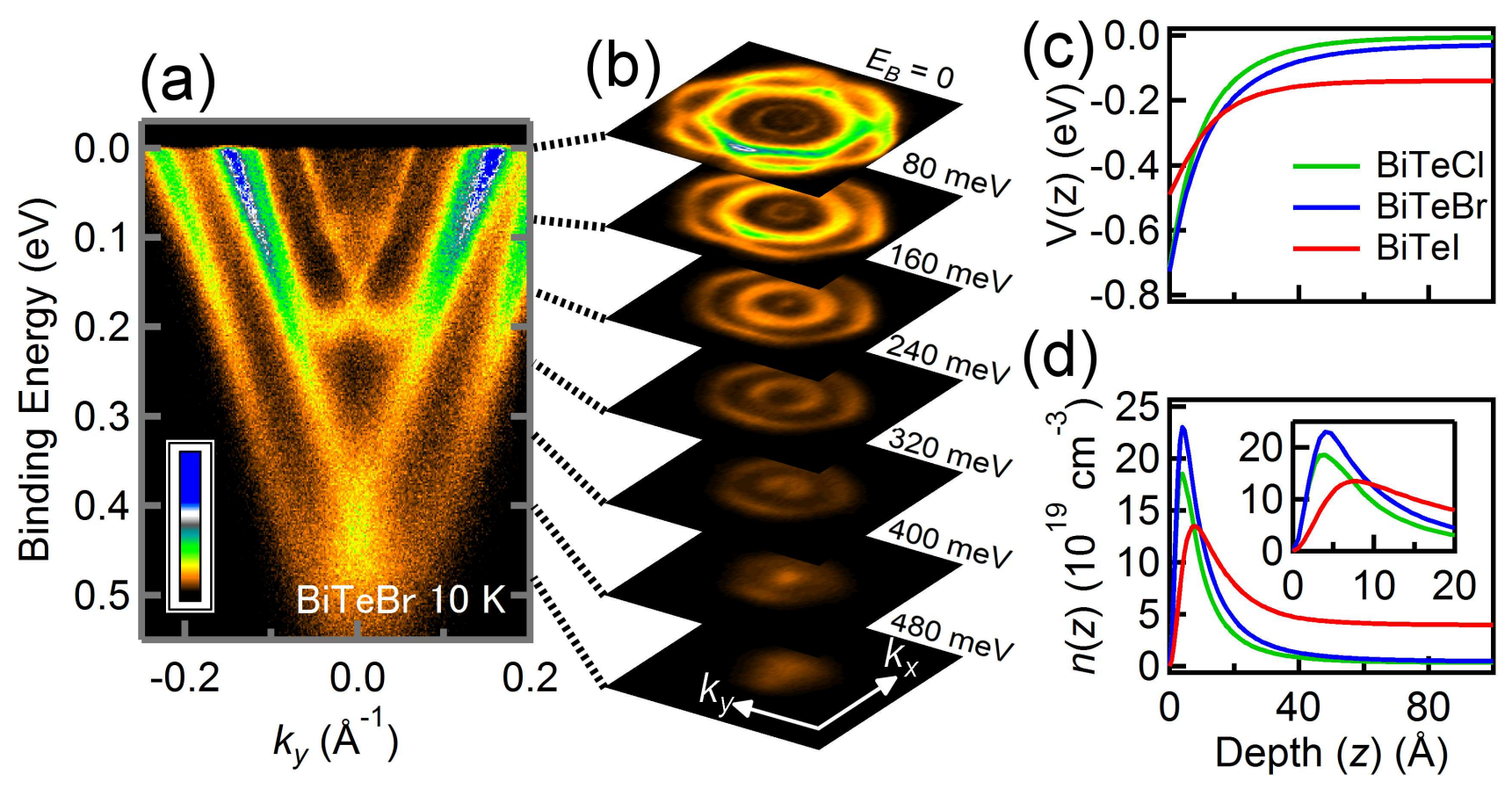

Figure 2 LY13668 05Feb2013 

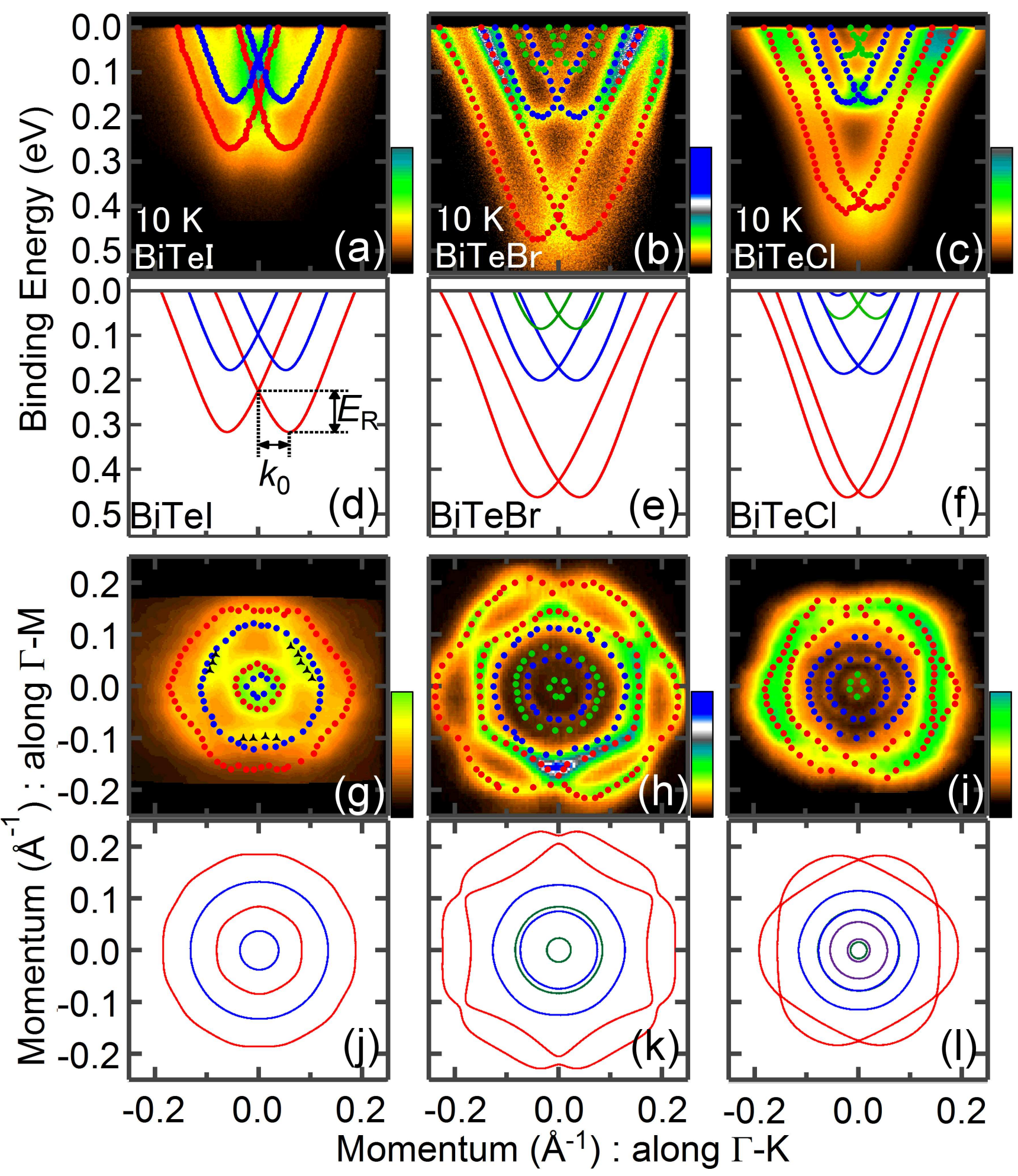


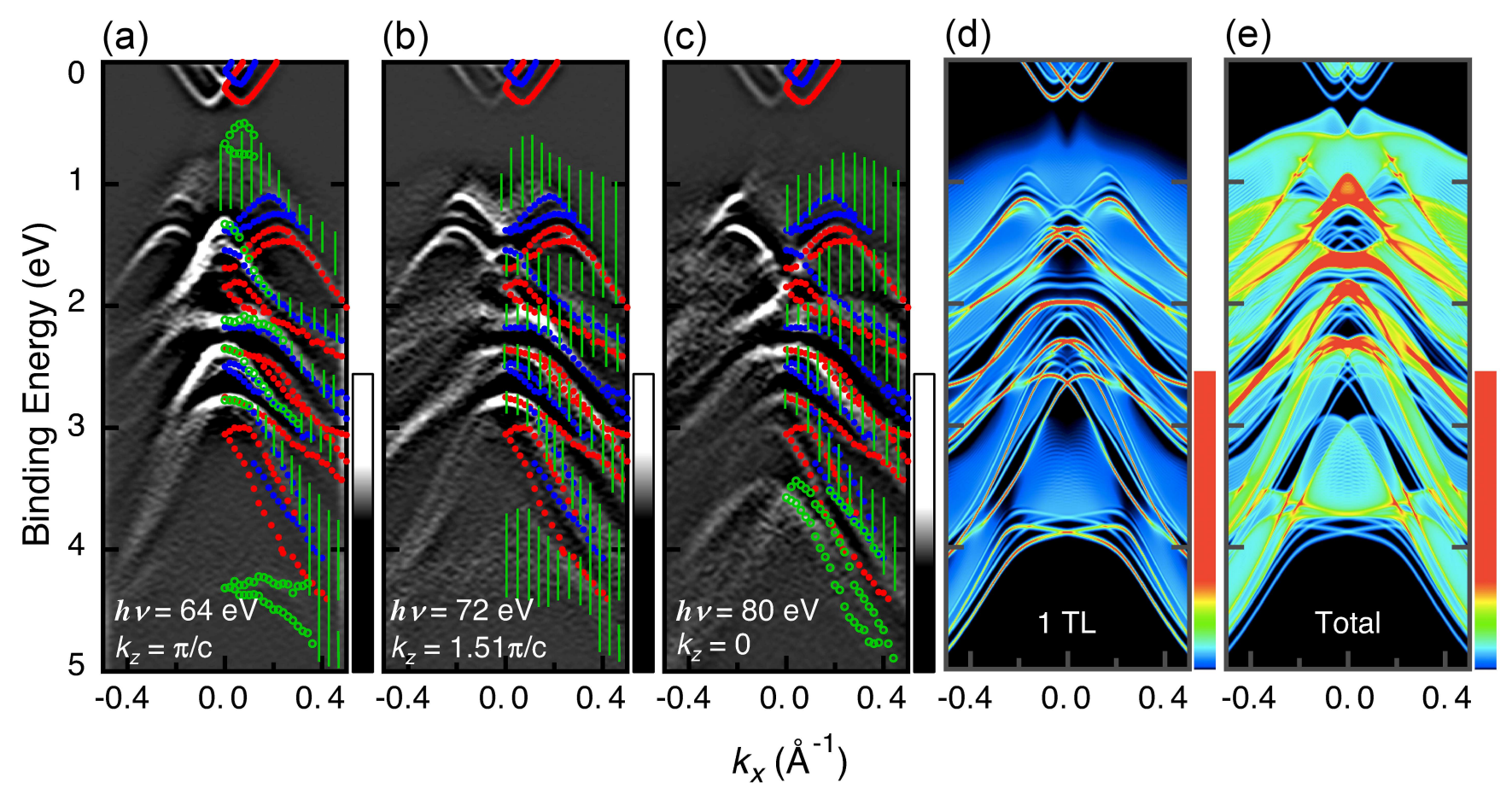

Figure $4 \quad$ LY13668 05Feb2013 